\title{
Use and misuse of temperature normalisation in meta- analyses of thermal responses of biological traits
}

\author{
Dimitrios - Georgios Kontopoulos ${ }^{\text {Corresp., }}{ }^{1,2}$, Bernardo García-Carreras ${ }^{2}$, Sofía Sal ${ }^{2}$, Thomas P. Smith ${ }^{2}$, \\ Samraat Pawar ${ }^{2}$ \\ ${ }^{1}$ Science and Solutions for a Changing Planet DTP, Imperial College London, London, United Kingdom \\ 2 Department of Life Sciences, Silwood Park, Imperial College London, Ascot, Berkshire, United Kingdom \\ Corresponding Author: Dimitrios - Georgios Kontopoulos \\ Email address: d.kontopoulos13@imperial.ac.uk
}

There is currently unprecedented interest in quantifying variation in thermal physiology among organisms, especially in order to understand and predict the biological impacts of climate change. A key parameter in this quantification of thermal physiology is the performance or value of a rate, across individuals or species, at a common temperature (temperature normalisation). An increasingly popular model for fitting thermal performance curves to data - the Sharpe-Schoolfield equation - can yield strongly inflated estimates of temperature-normalised rate values. These deviations occur whenever a key thermodynamic assumption of the model is violated, i.e. when the enzyme governing the performance of the rate is not fully functional at the chosen reference temperature. Using data on 1,758 thermal performance curves across a wide range of species, we identify the conditions that exacerbate this inflation. We then demonstrate that these biases can compromise tests to detect metabolic cold adaptation, which requires comparison of fitness or rate performance of different species or genotypes at some fixed low temperature. Finally, we suggest alternative methods for obtaining unbiased estimates of temperature-normalised rate values for meta-analyses of thermal performance across species in climate change impact studies. 


\title{
Use and misuse of temperature normalisation in
}

\section{meta-analyses of thermal responses of biological traits}

\author{
Dimitrios - Georgios Kontopoulos ${ }^{1,2, *}$ \\ Bernardo García-Carreras ${ }^{2}$ \\ Sofía $\mathrm{Sal}^{2}$ \\ Thomas P. Smith ${ }^{2}$ \\ Samraat Pawar $^{2}$
}

1. Science and Solutions for a Changing Planet DTP;

2. Department of Life Sciences, Imperial College London, Silwood Park, Ascot, Berkshire SL5 6 7PY, UK;

* Corresponding author; e-mail: d.kontopoulos13@imperial.ac.uk. 


\begin{abstract}
There is currently unprecedented interest in quantifying variation in thermal physiology among organisms, especially in order to understand and predict the biological impacts of climate change. A key parameter in this quantification of thermal physiology is the performance
\end{abstract}

\section{Introduction}

Temperature is a key factor that directly or indirectly governs the performance of biochemical re- 
(MCA; e.g., see Seibel et al. 2007; White et al. 2012; Clarke 2017), according to which coldadapted individuals exhibit higher metabolic rates at low temperatures (well below $T_{\mathrm{pk}}$; see fig. 1) than individuals adapted to higher temperatures.

The TPCs of fundamental biological rates (traits) are generally unimodal, and biological rate versus temperature relationships are typically well-described by mathematical models that quantify four key features of the response: the temperature where the performance peaks $\left(T_{\mathrm{pk}}\right)$, the rate performance at a reference temperature $\left(B_{0}\right)$, typically well below $T_{\mathrm{pk}}$ within its operational temperature range (Pawar et al. 2016), the rise of the rate up to $T_{\mathrm{pk}}(E)$, and the fall after $T_{\mathrm{pk}}\left(E_{\mathrm{D}}\right)$ (fig. 1). The normalised rate value $B_{0}$ is particularly important, as it allows rate performance to be standardised for comparison across individuals and species (Gillooly et al. 2001). In particular, the inference of normalised rate values at a reference temperature between species is key for studying MCA, or for comparisons of the performance of different biological rates (e.g., photosynthesis and respiration) at a common temperature (e.g., see Padfield et al. 2017).

Partly mechanistic models that explicitly link a cellular, organismal, or population rate's value to the temperature-dependence of the underlying biochemical kinetics (e.g., Johnson and Lewin 1946; Sharpe and DeMichele 1977; Schoolfield et al. 1981; Ikemoto 2005; Corkrey et al. 2012; Hobbs et al. 2013; DeLong et al. 2017) are becoming increasingly popular for quantifying empirically observed TPCs (Hochachka and Somero 2002). Such models have occasionally received criticism on the grounds that they only constitute phenomenological statistical descriptions, as their assumptions are too simplistic and cannot be directly mapped onto physiological or ecological rates, which should be driven by a far more complex interplay of processes (e.g., Clarke 2004; Clarke and Fraser 2004; Clarke 2006, 2017; but see Gillooly et al. 2006). Nevertheless, these models continue to be used in the literature as they can adequately fit a large variety of experimentally determined TPCs, enabling the quantification of various aspects of the shape of the performance curve.

Among these models, the Sharpe-Schoolfield model (Schoolfield et al. 1981) has been frequently used in recent studies to address both ecological and evolutionary questions about the 
effects of temperature change on individuals, populations, and communities (Barmak et al. 2014; Barneche et al. 2014; Fand et al. 2014; Simoy et al. 2015; Barneche et al. 2016; Padfield et al.

\section{Theoretical context}

The Sharpe-Schoolfield model proposes that the effect of temperature on the performance of a biological rate largely reflects the thermal sensitivity of a single rate-limiting enzyme that becomes deactivated at both extreme-high and low temperatures (Schoolfield et al. 1981). Nevertheless, 
low temperature inactivation is hard to detect, possibly because it requires multiple rate measurements at low temperatures for inferring accurate parameter estimates (see Pawar et al. 2016). Such resolution is typically lacking in currently available datasets of thermal performance. For this reason, it is most often more parsimonious to use a simpler version of the full model that ignores low-temperature enzyme inactivation (fig. 1):

$$
B(T)=B_{0} \cdot \frac{e^{\frac{-E}{k} \cdot\left(\frac{1}{T}-\frac{1}{T_{\mathrm{ref}}}\right)}}{1+e^{\frac{E_{\mathrm{D}}}{k} \cdot\left(\frac{1}{T_{\mathrm{h}}}-\frac{1}{T}\right)}} .
$$

Here, $B$ is the value of the rate at a given temperature $T(\mathrm{~K}), E$ is the activation energy $(\mathrm{eV})$, which controls the rise of the curve up to the peak, $E_{\mathrm{D}}$ is the de-activation energy $(\mathrm{eV})$, which sets the rate at which the rate falls after the peak, $T_{\mathrm{h}}(\mathrm{K})$ is the temperature at which $50 \%$ of the enzyme units are inactive, and $k$ is the Boltzmann constant $\left(8.617 \cdot 10^{-5} \mathrm{eV} \cdot \mathrm{K}^{-1}\right) . B_{0}$ is the value of the rate at a reference (normalisation) temperature $T_{\text {ref }}$-i.e., $B_{0} \approx B\left(T_{\text {ref }}\right)$ - assuming enzyme units are fully operational at that temperature. The model can also be reformulated without normalisation, but then $B_{0}$ would lose any biological meaning (see section A2.1 in Appendix A). The assumption of this model variant is that, at low temperatures, the population of the key enzyme remains fully active, with low rate performance values being driven by the decreased amount of kinetic energy which causes biochemical reactions to proceed at a very low rate.

Schoolfield et al. (1981) originally suggested using $T_{\text {ref }}=25^{\circ} \mathrm{C}$, a choice they considered appropriate for most poikilotherm species. This suggestion has frequently been followed (see table A1 and fig. A1 in Appendix A). However, when non-negligible loss of enzyme activity occurs at $T_{\text {ref }}-$ e.g., due to denaturation or inactivation of some other component of the metabolic pathway $-B_{0}$ overestimates the real value of the rate at that temperature $\left(B\left(T_{\text {ref }}\right)\right)$ (Ikemoto 2005$)$. This is particularly problematic for comparisons of $B_{0}$ across diverse species, as significant temperaturemediated inactivation may begin at very different temperatures, potentially leading to different degrees of inaccuracy in the $B_{0}$ estimates. 


\section{The inflation of rate value at reference temperature $\left(B_{0}\right)$}

We first consider why $B_{0}$ can be biased. For this, in addition to the parameters in eq. (1) $\left(B_{0}, E\right.$, $E_{\mathrm{D}}, T_{\mathrm{h}}, T_{\text {ref }}$ ), two extra parameters need to be defined to capture all aspects of the shape of the TPC: the temperature at which the TPC peaks $\left(T_{\mathrm{pk}}\right)$, and the performance at that peak $\left(P_{\mathrm{pk}}\right.$; see sections A2.2-3 in Appendix A for their derivations). Setting $T=T_{\text {ref }}$ in eq. (1) shows that the amount by which $B_{0}$ will deviate from $B\left(T_{\text {ref }}\right)$ is equal to the denominator of eq. (1):

$$
B\left(T_{\mathrm{ref}}\right)=B_{0} \cdot \frac{1}{1+e^{\frac{E_{\mathrm{D}}}{k} \cdot\left(\frac{1}{T_{\mathrm{h}}}-\frac{1}{T_{\mathrm{ref}}}\right)}}
$$

When $T_{\text {ref }}$ is much lower than $T_{\mathrm{h}}$ (the temperature at which $50 \%$ of the enzyme units become inactive), $B_{0} \approx B\left(T_{\text {ref }}\right)$ because the denominator $\approx 1$. On the other hand, as the chosen $T_{\text {ref }}$ approaches $T_{\mathrm{h}}$ - or exceeds it,$- B_{0}$ will increasingly deviate from $B\left(T_{\text {ref }}\right)$. In any case, $B_{0}$ will always be greater than $B\left(T_{\text {ref }}\right)$ (at best, by a negligible amount) because of the denominator of eq. (2). To explore this behaviour numerically across real TPCs of a single biological rate (for consistency reasons), we compiled a dataset of phytoplankton growth rates versus temperature (a combination of the López-Urrutia et al. 2006, Rose and Caron 2007, Bissinger et al. 2008, and Thomas et al. 2012 datasets), containing 672 species/strains with growth rate being measured at multiple temperatures per species/strain. To each TPC in this dataset, we fitted the SharpeSchoolfield model across a range of $T_{\text {ref }}$ values $\left(-10^{\circ} \mathrm{C}\right.$ to $\left.30^{\circ} \mathrm{C}\right)$ using the nonlinear least-squares method (Levenberg-Marquardt algorithm). In order to eliminate less reliable fitted parameter estimates, we rejected fits with i) an $R^{2}$ below 0.5 (raising this cutoff to 0.9 yielded qualitatively identical results) or ii) fewer than four data points either before or after $T_{\mathrm{pk}}$. Based on these criteria, the number of accepted fits per $T_{\text {ref }}$ value ranged from 121 to 126 out of 672 starting TPCs (for an $R^{2}$ cutoff of 0.5 ). The variation in the number of retained parameter estimates is due to the different $T_{\text {ref }}$ values that we used which can cause small changes in the quality of the fit, leading to the occasional exclusion of some fits with $R^{2}$ values very close to the cutoff. The 

the data.

computer code - along with the names and versions of all modules or packages used - for the main analyses of this study (including fitting the Sharpe-Schoolfield model to TPCs) is available at https://github.com/dgkontopoulos/Kontopoulos_et_al_temperature_normalisation_2017.

\section{Identification of conditions that lead to a severely overestimated $B_{0}$}

We next determine the characteristics of TPCs (parameter combinations of the Sharpe-Schoolfield model) that lead to a severely overestimated $B_{0}$. This is a complex problem and not just a matter of determining the difference between $T_{\mathrm{h}}$ and $T_{\mathrm{ref}}$, because the denominator of eq. (2) also includes the $E_{\mathrm{D}}$ parameter. As $E_{\mathrm{D}}$ influences the relationship between $T_{\mathrm{h}}$ and $T_{\mathrm{pk}}$ (see section $\mathrm{A} 2.2$ in Appendix A), it is necessary to take into account the interplay of $T_{\mathrm{h}}$ and $T_{\mathrm{ref}}$ with $T_{\mathrm{pk}}$. To address this, we use a conditional inference tree (a machine learning algorithm; Hothorn et al. 2006) to determine the TPC model's parameter combinations that lead to strong overestimation.

For maximising the power of the machine learning method we used a larger dataset — a subset of the Biotraits database (a substantial collection of performance measurements of ecological traits and physiological rates at multiple temperatures from a wide range of species; Dell et al. 2013) combined with additional data extracted from the published literature (see section A5 in Appendix A). We first fitted the Sharpe-Schoolfield model to each empirical TPC in this dataset. As the dataset is very diverse - including, among others, rates from bacteria, macroalgae, and terrestrial plants - we set $T_{\text {ref }}$ to $0^{\circ} \mathrm{C}$ so that we could obtain reasonable estimates (i.e., at a temperature below $\left.T_{\mathrm{pk}}\right)$ of $B_{0}$ and $B\left(T_{\mathrm{ref}}\right)$ even for cold-adapted species with low $T_{\mathrm{pk}}$ values. It is worth stressing that such a low $T_{\text {ref }}$ value is indeed appropriate because, as mentioned in the "Theoretical context" section, experimentally determined TPCs generally do not possess the required resolution for detecting low-temperature enzyme inactivation. Thus, it is safe to assume that rate estimates will be reasonable at low temperatures, even at $0^{\circ} \mathrm{C}$. In total, 1,758 species/individual curves were produced from this dataset. We did not filter the results based on goodness of fit metrics because we are interested in all the different parameter combinations regardless of how well they describe 
We then analysed this ensemble of fitted curves through the construction of a conditional inference tree from the data (see section A3.1 in Appendix A for details). More precisely, we specified a binary response variable: $B_{0}$ is above or below $P_{\mathrm{pk}}$. The choice of $P_{\mathrm{pk}}$ as the cutoff was due to the very high classification performance of the resulting model, especially when compared to other possible cutoffs (e.g., a three-fold increase from $B\left(T_{\text {ref }}\right)$ ) which performed poorly. The predictor variables were the differences between i) $T_{\mathrm{pk}}$ and $T_{\mathrm{h}}$, ii) $T_{\mathrm{pk}}$ and $T_{\mathrm{ref}}$, and iii) $T_{\mathrm{h}}$ and $T_{\text {ref }}$ for each fit. The model was constrained by setting the maximum allowed $p$-value at each internal node below $10^{-10}$. Its performance was evaluated with the Matthews correlation coefficient (MCC; Matthews 1975), a metric often used for machine learning models with a binary response. This metric takes values from -1 (complete disagreement with data) to 1 (complete agreement with data) and is considered reliable even when the different response states of the model (in this case $B_{0}>P_{\mathrm{pk}}$ and $\left.B_{0}<P_{\mathrm{pk}}\right)$ are not evenly sampled. To further ensure that the model was accurate and generalisable, we also estimated its performance against a distinct dataset of 405 TPCs (testing dataset). The data for these curves were also part of the Biotraits database - similarly to the 1,758 curves - but were not used for training the model.

\section{Implications of the inflation for investigations of thermal adaptation}

Among other ecological and evolutionary questions, the effects of adaptation to different thermal environments on the shape of the TPC (e.g., see Huey and Kingsolver 1989; Angilletta et al. 2003; Angilletta 2009; Angilletta et al. 2010; Clarke 2017) can be investigated using estimates from the Sharpe-Schoolfield model. For example, a study may aim to uncover whether there are any trade-offs between performance at lower and higher temperatures by correlating $B_{0}$ and $T_{\mathrm{pk}}(\mathrm{e} . \mathrm{g}$., a negative correlation would suggest that high performance at warmer temperatures would come at the cost of lower performance at colder temperatures). Overestimating $B_{0}$ - especially for coldadapted species with a $T_{\mathrm{h}}$ value close to $T_{\text {ref }}$ - may potentially introduce such correlations where none existed, serving as false-positive evidence for the MCA hypothesis.

To explore this possible issue, we generated a synthetic dataset of 1,000 negatively skewed 

correlation between the normalised rate values $\left(B_{0}\right.$ and $\left.B\left(T_{\mathrm{ref}}\right)\right)$ and the corresponding $T_{\mathrm{pk}}$ values. 


\section{Results}

\section{Conditions that lead to different degrees of inflation of $B_{0}$ estimates}

Using the phytoplankton growth rates dataset, we show that, contingent on the difference between $T_{\mathrm{h}}$ and $T_{\text {ref }}, B_{0}$ can be considerably greater than $B\left(T_{\text {ref }}\right)$ (fig. 2 ). More precisely, the deviation of $B_{0}$ from $B\left(T_{\text {ref }}\right)$ decreases nonlinearly with the difference between $T_{\text {ref }}$ and $T_{\mathrm{h}}$ (panel A). In many circumstances, the deviation of $B_{0}$ is extreme, becoming even greater than the rate value at or near optimum temperature, $P_{\mathrm{pk}}$ (panel B).

The search for thermal response parameter combinations that lead to $B_{0}$ being above $P_{\mathrm{pk}}$ (highly overestimated) or below it (less overestimated) resulted in a conditional inference tree with four terminal nodes (fig. 3). In each of those nodes, $B_{0}$ was nearly exclusively below or above $P_{\mathrm{pk}}$. This machine learning model exhibited high performance both on the training dataset $(\mathrm{MCC}=0.954)$ and the testing dataset $(\mathrm{MCC}=0.824$; section A3.2 in Appendix A). The sets of thermal response parameters in which $B_{0}$ was greater than $P_{\mathrm{pk}}$ almost always had either a $T_{\mathrm{h}}-T_{\text {ref }}$ difference that was less than 0.6 (relatively narrow curves), or a $T_{\mathrm{pk}}-T_{\text {ref }}$ difference of 49.1 or lower (relatively wide curves).

\section{Impacts of the overestimation of $B_{0}$ on tests for MCA}

In total, we were able to obtain thermal response parameter estimates for 968 simulated curves, as the nonlinear least-squares algorithm failed to converge on solutions for the remaining 32 . In the first test for MCA the distributions of $B_{0}$ estimates differed between the two groups $(D=0.18$, $p=1.7 \cdot 10^{-6}$ ), with species adapted to colder temperatures having a higher median value of $B_{0}$ (fig. $4 \mathrm{~A}$, light blue violin plots). In contrast, the two distributions of $B\left(T_{\text {ref }}\right)$ estimates were statistically indistinguishable $(D=0.07, p=0.21)$, as expected (fig. 4A, green violin plots). The overestimation of $B_{0}$ also affected the second MCA test, as a weak negative correlation between $B_{0}$ and $T_{\mathrm{pk}}$ was detected, but not between $B\left(T_{\mathrm{ref}}\right)$ and $T_{\mathrm{pk}}$ (fig. $4 \mathrm{~B}, \mathrm{C}$ ). These results indicate that the inflation of $B_{0}$ can provide false support for the MCA hypothesis, even for datasets with complete 
absence of this pattern.

\section{Discussion}

In this paper we have addressed the consequences of estimating the value of a rate at a reference temperature, $B_{0}$, using the Sharpe-Schoolfield model, but without satisfying one of its fundamental assumptions: that the key enzyme - which is responsible for the temperature dependence of the rate - is fully functional at the reference temperature. When this assumption is not met, $B_{0}$ will overestimate the real rate performance at the reference temperature, $B\left(T_{\text {ref }}\right)$ (Ikemoto, 2005).

We explain how the discrepancy between $B_{0}$ and $B\left(T_{\text {ref }}\right)$ arises and determine the conditions under which it becomes particularly pronounced using a machine learning approach (fig. 3). The resulting conditional inference tree shows that $B_{0}$ estimates will generally exceed the rate performance at the peak of the curve $\left(P_{\mathrm{pk}}\right)$ as long as: i) $T_{\mathrm{pk}}-T_{\mathrm{h}}$ is less than $\sim 37.58^{\circ} \mathrm{C}$ and $T_{\mathrm{h}}-T_{\text {ref }}$ is less than $\sim 0.6^{\circ} \mathrm{C}$, or ii) $T_{\mathrm{pk}}-T_{\mathrm{h}}$ is greater than $\sim 37.58^{\circ} \mathrm{C}$ and $T_{\mathrm{pk}}-T_{\text {ref }}$ is less than $\sim 49.11^{\circ} \mathrm{C}$. In any other case, $B_{0}$ would most likely be smaller than $P_{\mathrm{pk}}$, although its inflation may well still be of concern. Using a synthetic dataset, we then demonstrate that wrongly assuming $B_{0}=B\left(T_{\text {ref }}\right)$ can lead to erroneous conclusions in analyses of thermal adaptation, as the overestimation of $B_{0}$ can mimic the effects of metabolic cold adaptation (fig. 4) (a Type I error).

It is important to note that while we focus on the four-parameter version of the Sharpe-Schoolfield model in this study, the inflation of $B_{0}$ estimates also mathematically occurs in the variant of the model that assumes enzyme inactivation at both high and low temperatures. Thus, caution is warranted regardless of the model variant that is chosen. Beyond this issue, fitting the simpler model instead of its full counterpart may potentially give rise to other inherent biases but, to our knowledge, a thorough comparison of the two model variants across different organismal groups and rates is not available.

255 As mentioned before, previous studies have tended to set the $T_{\text {ref }}$ - usually at a value of $25^{\circ} \mathrm{C}$ - while fitting the Sharpe-Schoolfield model without considering the potential inflation of $B_{0}$ (ta- 
ble A1 and fig. A1, Appendix A). Whether results of these studies have been compromised by an inappropriate use of $T_{\text {ref }}$ is impossible to determine definitively because most of these studies report either $T_{\mathrm{h}}$ or $T_{\mathrm{pk}}$ estimates, whereas the machine learning model depends on both (see the 'Conditions leading to a severely overestimated $B_{0}$ ' section), along with the value of $T_{\text {ref. If these }}$ data were available, using the machine learning model that we generated would provide a straightforward procedure to identify cases where $B_{0}$ is highly likely to be extremely overestimated (i.e, greater than $\left.P_{\mathrm{pk}}\right)$. In fact, the only study where all necessary parameter estimates were reported for all fitted curves was that by Padfield et al. (2016). In that study, the maximum difference of $T_{\mathrm{h}}$ from $T_{\mathrm{pk}}$ is $2.49^{\circ} \mathrm{C}$, and the minimum difference of $T_{\text {ref }}$ from $T_{\mathrm{h}}$ is $5.79^{\circ} \mathrm{C}$, which, according to the machine learning model (see fig. 3), are sufficient for the $B_{0}$ estimates to be below those of $P_{\mathrm{pk}}$. Having said that, as we showed in this paper, the fact that the overestimation of $B_{0}$ is not extreme does not necessarily rid any drawn conclusions of bias (e.g., the possibility of falsely detecting the effect of MCA).

In any case, it is crucial to point out that choosing an appropriate reference temperature (i.e., one that is low enough but within the temperature range that the species can endure) is not - on its own - a sufficient strategy to avoid the overestimation of $B_{0}$. As different species or individuals will most likely not share a common $T_{\mathrm{h}}$ value, the difference between $T_{\mathrm{h}}$ and $T_{\text {ref }}$ will vary across the dataset (see fig. 2). This approach could again lead to an exaggeration (which may however be very small) of some $B_{0}$ estimates and is therefore not an elegant solution to the problem.

\section{Comparisons of temperature-normalised rates of diverse species}

\section{When data span the entire TPC}

For studies in which the end goal is to compare the performance of different species at a common temperature, the simplest approach would be to fit the Sharpe-Schoolfield model - with or without normalising $B_{0}$ at a reference temperature - and compare estimates of $B\left(T_{\text {ref }}\right)$, calculated a posteriori. The confidence intervals around $B\left(T_{\text {ref }}\right)$ can then be estimated by bootstrapping. Another option to avoid the issue of rate overestimation is to consider fitting other models, such as the 
macromolecular rates model (Hobbs et al. 2013) or the enzyme-assisted Arrhenius model (DeLong et al. 2017).

When data only cover the rising part of the TPC

While the previous solutions are applicable to thermal response datasets that capture either the rise of the curve or its entirety, few studies report temperature performance measurements after the unimodal peak of the response (Dell et al., 2011). Therefore, to obtain an estimate of baseline performance from a dataset that only covers the exponential rise component, one could instead fit the Boltzmann-Arrhenius model (e.g., see Gillooly et al. 2001),

$$
B(T)=B_{0} \cdot e^{\frac{-E}{k} \cdot\left(\frac{1}{T}-\frac{1}{T_{\mathrm{ref}}}\right)},
$$

which does not suffer from the problems of the Sharpe-Schoolfield model, as $B\left(T_{\text {ref }}\right)$ indeed simplifies to $B_{0}$.

A second alternative model is the one that includes the $Q_{10}$ factor (see Gillooly et al. 2001), i.e. the rate of change in biological rate performance after a temperature rise of $10^{\circ} \mathrm{C}$ :

$$
Q_{10}=\left(\frac{B\left(T_{2}\right)}{B\left(T_{1}\right)}\right)^{\frac{10}{T_{2}-T_{1}}}
$$

In this case, one would first estimate the value of $Q_{10}$ from known rate values at two temperatures, and use it to calculate the rate value at the reference temperature:

$$
B\left(T_{\text {ref }}\right)=B\left(T_{1}\right) \cdot Q_{10}^{\frac{T_{\text {ref }}-T_{1}}{10}} .
$$

Regardless of which of these two models is chosen, careful attention must be paid to ensure that the biological rate increases exponentially across the entire temperature range, without signs 
of a plateau being reached. Otherwise, the estimates may yet again be biased.

\section{Using the 'intrinsic optimum temperature' instead of $T_{\text {ref }}$}

Alternatively, baseline performance could be defined as the height of the curve at the temperature where the population of the key enzyme is fully active, which should be characteristic for each individual or species. In the Sharpe-Schoolfield model, the denominator indicates the percentage of enzymes that are active. Therefore, in the four-parameter variant of the model, the intrinsic optimum temperature could be estimated as the highest temperature at which this percentage is sufficiently high (e.g., at 99\%). If, instead, the model of choice is the Sharpe-Schoolfield variant that also accounts for enzyme inactivation at low temperatures, there will be a unique temperature at which the enzyme population is $100 \%$ active. Otherwise, the intrinsic optimum temperature can also be obtained from the Sharpe-Schoolfield-Ikemoto (SSI) model (Ikemoto 2005). This model integrates the law of total effective temperature - often used in studies of arthropod or parasite development - within the Sharpe-Schoolfield model, replacing $T_{\text {ref }}$ with the intrinsic optimum temperature. However, this model introduces an extra parameter and is more challenging to fit compared to the original Sharpe-Schoolfield model. To mitigate this problem, software implementations have been developed that reduce the computation time from often more than 3 hours (Ikemoto 2008) down to less than a second (Shi et al. 2011; Ikemoto et al. 2013).

\section{Conclusions}

Obtaining accurate estimates of temperature-normalised rate performance is of crucial importance - especially in the face of climate change - for comparisons of the same rate across different organisms, or different rates within an individual. In this context, our study explains why temperaturenormalised rate estimates obtained using the Sharpe-Schoolfield model can be strongly exaggerated - in comparison to the true rate values - when one of the assumptions of the model is violated, and gives an example of possible consequences of this exaggeration. The suggestions that we pro- 
vide to address this issue should be useful to the burgeoning studies on ectotherm thermal performance and climate change, both for performing meta-analyses and for determining appropriate temperature ranges in laboratory experiments. 


\section{References}

Angilletta, M. J. 2009. Thermal adaptation: a theoretical and empirical synthesis. Oxford University Press.

Angilletta, M. J., R. B. Huey, and M. R. Frazier. 2010. Thermodynamic effects on organismal performance: is hotter better? Physiological and Biochemical Zoology 83:197-206.

Angilletta, M. J., R. S. Wilson, C. A. Navas, and R. S. James. 2003. Tradeoffs and the evolution of thermal reaction norms. Trends in Ecology \& Evolution 18:234-240.

Barmak, D. H., C. O. Dorso, M. Otero, and H. G. Solari. 2014. Modelling interventions during a dengue outbreak. Epidemiology and Infection 142:545-561.

Barneche, D., M. Kulbicki, S. Floeter, A. Friedlander, and A. Allen. 2016. Energetic and ecological constraints on population density of reef fishes. Proceedings of the Royal Society of London. Series B, Biological sciences 283:20152186.

Barneche, D. R., M. Kulbicki, S. R. Floeter, A. M. Friedlander, J. Maina, and A. P. Allen. 2014. Scaling metabolism from individuals to reef-fish communities at broad spatial scales. Ecology Letters 17:1067-1076.

Bissinger, J. E., D. J. Montagnes, D. Atkinson, et al. 2008. Predicting marine phytoplankton maximum growth rates from temperature: Improving on the Eppley curve using quantile regression. Limnology and Oceanography 53:487-493.

Brown, J. H., J. F. Gillooly, A. P. Allen, V. M. Savage, and G. B. West. 2004. Toward a metabolic theory of ecology. Ecology 85:1771-1789.

Clarke, A. 2004. Is there a universal temperature dependence of metabolism? Functional Ecology $18: 252-256$.

- 2006. Temperature and the metabolic theory of ecology. Functional Ecology 20:405-412. 
- 2017. Principles of Thermal Ecology: Temperature, Energy, and Life. Oxford University Press.

Clarke, A., and K. Fraser. 2004. Why does metabolism scale with temperature? Functional Ecology 18:243-251.

Corder, G. W., and D. I. Foreman. 2014. Nonparametric Statistics: A Step-by-Step Approach, 2nd Edition. John Wiley \& Sons.

Corkrey, R., J. Olley, D. Ratkowsky, T. McMeekin, and T. Ross. 2012. Universality of thermodynamic constants governing biological growth rates. PLoS One 7:e32003.

Dell, A. I., S. Pawar, and V. M. Savage. 2011. Systematic variation in the temperature dependence of physiological and ecological traits. Proceedings of the National Academy of Sciences 108:10591-10596.

2013. The thermal dependence of biological traits. Ecology 94:1205-1206.

DeLong, J. P., J. P. Gibert, T. M. Luhring, G. Bachman, B. Reed, A. Neyer, and K. Montooth. 2017. The combined effects of reactant kinetics and enzyme stability explain the temperature dependence of metabolic rates. Ecology and Evolution .

Fand, B. B., H. E. Z. Tonnang, M. Kumar, A. L. Kamble, and S. K. Bal. 2014. A temperaturebased phenology model for predicting development, survival and population growth potential of the mealybug, Phenacoccus solenopsis Tinsley (Hemiptera: Pseudococcidae). Crop Protection 55:98-108.

Gillooly, J., A. Allen, V. Savage, E. Charnov, G. West, and J. Brown. 2006. Response to Clarke and Fraser: effects of temperature on metabolic rate. Functional Ecology 20:400-404.

Gillooly, J. F., J. H. Brown, G. B. West, V. M. Savage, and E. L. Charnov. 2001. Effects of size and temperature on metabolic rate. Science 293:2248-2251. 
Johnson, Z. I. 2006. Niche Partitioning Among Prochlorococcus Ecotypes Along Ocean-Scale Environmental Gradients. Science 311:1737-1740. rates. ACS Chemical Biology 8:2388-2393.

Hochachka, P. W., and G. N. Somero. 2002. Biochemical Adaptation: Mechanism and Process in Physiological Evolution. Oxford University Press.

Hoffmann, A. A., and C. M. Sgrò. 2011. Climate change and evolutionary adaptation. Nature 470:479-485.

Hopp, M. J., and J. A. Foley. 2001. Global-scale relationships between climate and the dengue fever vector, Aedes aegypti. Climatic Change 48:441-463.

Hothorn, T., K. Hornik, and A. Zeileis. 2006. Unbiased recursive partitioning: a conditional inference framework. Journal of Computational and Graphical Statistics 15:651-674.

Huey, R. B., and J. G. Kingsolver. 1989. Evolution of thermal sensitivity of ectotherm performance. Trends in Ecology \& Evolution 4:131-135.

Ikemoto, T. 2005. Intrinsic optimum temperature for development of insects and mites. Environmental Entomology 34:1377-1387.

- 2008. Tropical malaria does not mean hot environments. Journal of Medical Entomology 45:963-969.

Ikemoto, T., I. Kurahashi, and P.-J. Shi. 2013. Confidence interval of intrinsic optimum temperature estimated using thermodynamic SSI model. Insect science 20:420-428.

Johnson, F. H., and I. Lewin. 1946. The growth rate of E. coli in relation to temperature, quinine and coenzyme. Journal of Cellular and Comparative Physiology 28:47-75.

Hobbs, J. K., W. Jiao, A. D. Easter, E. J. Parker, L. A. Schipper, and V. L. Arcus. 2013. Change in heat capacity for enzyme catalysis determines temperature dependence of enzyme catalyzed 
López-Urrutia, Á., E. San Martin, R. P. Harris, and X. Irigoien. 2006. Scaling the metabolic balance of the oceans. Proceedings of the National Academy of Sciences 103:8739-8744.

Matthews, B. W. 1975. Comparison of the predicted and observed secondary structure of T4 phage lysozyme. Biochimica et Biophysica Acta (BBA)-Protein Structure 405:442-451.

Padfield, D., C. Lowe, A. Buckling, R. Ffrench-Constant, Student Research Team, S. Jennings, F. Shelley, J. S. Ólafsson, and G. Yvon-Durocher. 2017. Metabolic compensation constrains the temperature dependence of gross primary production. Ecology Letters 20:1250-1260.

Padfield, D., G. Yvon-Durocher, A. Buckling, S. Jennings, and G. Yvon-Durocher. 2016. Rapid evolution of metabolic traits explains thermal adaptation in phytoplankton. Ecology Letters 19:133-142.

Pawar, S., A. I. Dell, and V. M. Savage. 2015. From metabolic constraints on individuals to the dynamics of ecosystems. Pages 3-36 in A. Belgrano, G. Woodward, and U. Jacob, eds. Aquatic Functional Biodiversity: An Ecological and Evolutionary Perspective. Elsevier.

Pawar, S., A. I. Dell, V. M. Savage, and J. L. Knies. 2016. Real versus artificial variation in the thermal sensitivity of biological traits. The American Naturalist 187:E41-E52.

Pörtner, H. O., A. F. Bennett, F. Bozinovic, A. Clarke, M. A. Lardies, M. Lucassen, B. Pelster, F. Schiemer, and J. H. Stillman. 2006. Trade-offs in thermal adaptation: The need for a molecular to ecological integration. Physiological and Biochemical Zoology 79:295-313.

Rose, J. M., and D. A. Caron. 2007. Does low temperature constrain the growth rates of heterotrophic protists? Evidence and implications for algal blooms in cold waters. Limnology and Oceanography 52:886-895.

Schoolfield, R., P. Sharpe, and C. Magnuson. 1981. Non-linear regression of biological temperature-dependent rate models based on absolute reaction-rate theory. Journal of Theoretical Biology 88:719-731. 
Schulte, P. M., T. M. Healy, and N. A. Fangue. 2011. Thermal performance curves, phenotypic plasticity, and the time scales of temperature exposure. Integrative and Comparative Biology 51:691-702.

Seibel, B. A., A. Dymowska, and J. Rosenthal. 2007. Metabolic temperature compensation and coevolution of locomotory performance in pteropod molluscs. Integrative and Comparative Biology 47:880-891.

Sharpe, P. J., and D. W. DeMichele. 1977. Reaction kinetics of poikilotherm development. Journal of Theoretical Biology 64:649-670.

Shi, P., T. Ikemoto, C. Egami, Y. Sun, and F. Ge. 2011. A modified program for estimating the parameters of the SSI model. Environmental Entomology 40:462-469.

Simoy, M. I., M. V. Simoy, and G. A. Canziani. 2015. The effect of temperature on the population dynamics of Aedes aegypti. Ecological Modelling 314:100-110.

Thomas, M. K., C. T. Kremer, C. A. Klausmeier, and E. Litchman. 2012. A global pattern of thermal adaptation in marine phytoplankton. Science 338:1085-1088.

Vimercati, L., S. Hamsher, Z. Schubert, and S. Schmidt. 2016. Growth of high-elevation Cryptococcus sp. during extreme freeze-thaw cycles. Extremophiles 20:579-588.

White, C. R., L. A. Alton, and P. B. Frappell. 2012. Metabolic cold adaptation in fishes occurs at the level of whole animal, mitochondria and enzyme. Proceedings of the Royal Society of London B: Biological Sciences 279:1740-1747.

Wohlfahrt, G., M. Bahn, E. Haubner, I. Horak, W. Michaeler, K. Rottmar, U. Tappeiner, and A. Cernusca. 1999. Inter-specific variation of the biochemical limitation to photosynthesis and related leaf traits of 30 species from mountain grassland ecosystems under different land use. Plant, Cell \& Environment 22:1281-1296. 


\section{Tables}

\begin{tabular}{cc}
\hline Parameter name & Estimation \\
\hline$\alpha$ & $\alpha \sim \mathscr{N}(\mu=10, \sigma=3)$ \\
$\beta$ & $\alpha-i, i \sim \mathscr{N}(\mu=4, \sigma=2)$ \\
Final curve width & original width $\cdot j, j \sim \mathscr{N}(\mu=25, \sigma=4)$ \\
Final curve height & original height $+k, k \sim \mathscr{N}(\mu=3, \sigma=0.8)$ \\
\hline
\end{tabular}

Table 1: Parameters for the generation of simulated curves. $\alpha$ and $\beta$ are shape parameters of the beta distribution, whereas the two other parameters generate variation in the width and the height of the curves. $\beta$ is constrained to be smaller than $\alpha$, in order for the resulting curves to be negatively skewed, similarly to the observed thermal response curves of biological rates. 


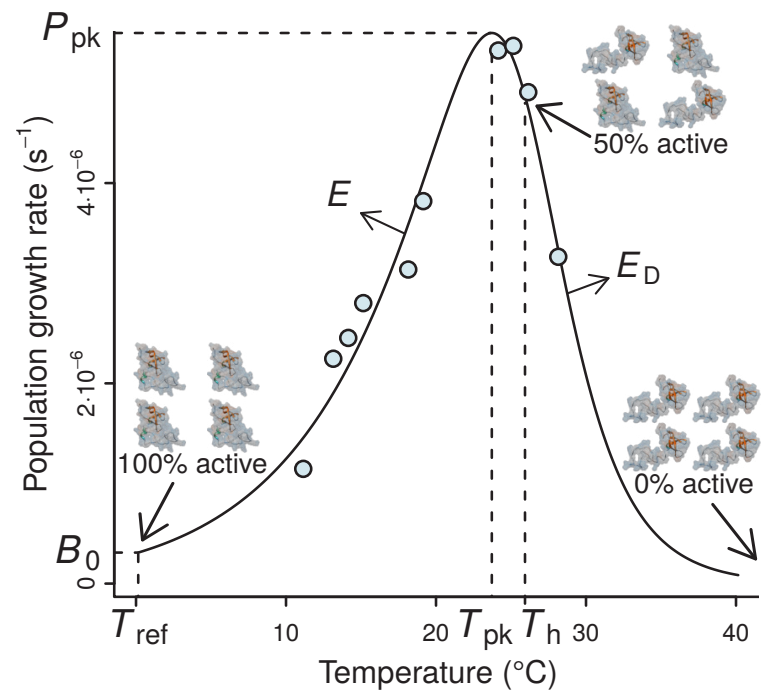

Figure 1: A typical example of the four-parameter Sharpe-Schoolfield model fitted to a thermal performance curve of Prochlorococcus marinus strain MIT9515 (Johnson 2006). As depicted, the model assumes that the activity of a single rate-controlling enzyme controls the apparent temperature dependence of the rate. $T_{\mathrm{h}}$ is defined as the temperature (before or after the peak) at which $50 \%$ of enzyme units are made inactive. Beyond $T_{\mathrm{h}}$, an increasing proportion of the enzyme population is deactivated, to the point where all of them become non-functional, and the curve falls to zero. $B_{0}$ accurately represents the real rate performance at a reference temperature $\left(T_{\text {ref }}\right)$, only if the enzyme population is fully functional at this particular $T_{\text {ref }}$, i.e., $T_{\text {ref }} \ll T_{\mathrm{h}}$; otherwise, $B_{0}$ will necessarily be greater than the real rate value at $T_{\text {ref }}\left(B\left(T_{\text {ref }}\right)\right)$. 

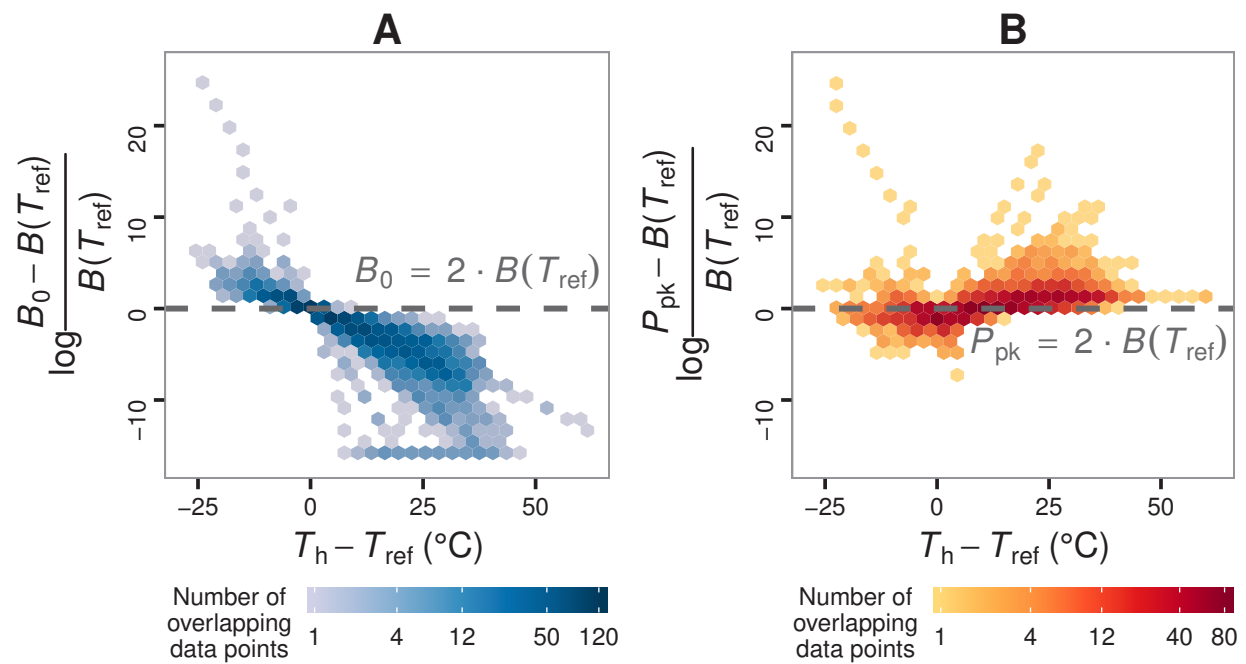

Figure 2: The effect of choice of reference temperature $T_{\text {ref }}$ on the deviation of $B_{0}$ from $B\left(T_{\text {ref }}\right)$ (panel A) and its relationship with $P_{\mathrm{pk}}($ panel B). The vertical axis of panel A stands for the logfold increase of $B_{0}$ from $B\left(T_{\text {ref }}\right)$, where a value of zero indicates that $B_{0}$ is double the real $B\left(T_{\text {ref }}\right)$ value. Zero is used here as a reference point around and above which $B_{0}$ becomes non-negligibly exaggerated. Data points were obtained by fitting the Sharpe-Schoolfield model to a dataset of phytoplankton growth rate measurements versus temperature (see main text) across a range of $T_{\text {ref }}$ values. The colour depth of each hexagon is proportional to the number of data points at that location in the graph. As expected from eq. (2), the deviation of $B_{0}$ from $B\left(T_{\text {ref }}\right)$ decreases nonlinearly with the difference between $T_{\mathrm{h}}$ and $T_{\text {ref }}$, to the point where the former asymptotically approaches zero (in linear scale). Towards the left end of the horizontal axis, the values of the estimates of $B_{0}$ even exceed those of the rate value at or close to optimum, $P_{\mathrm{pk}}$. 


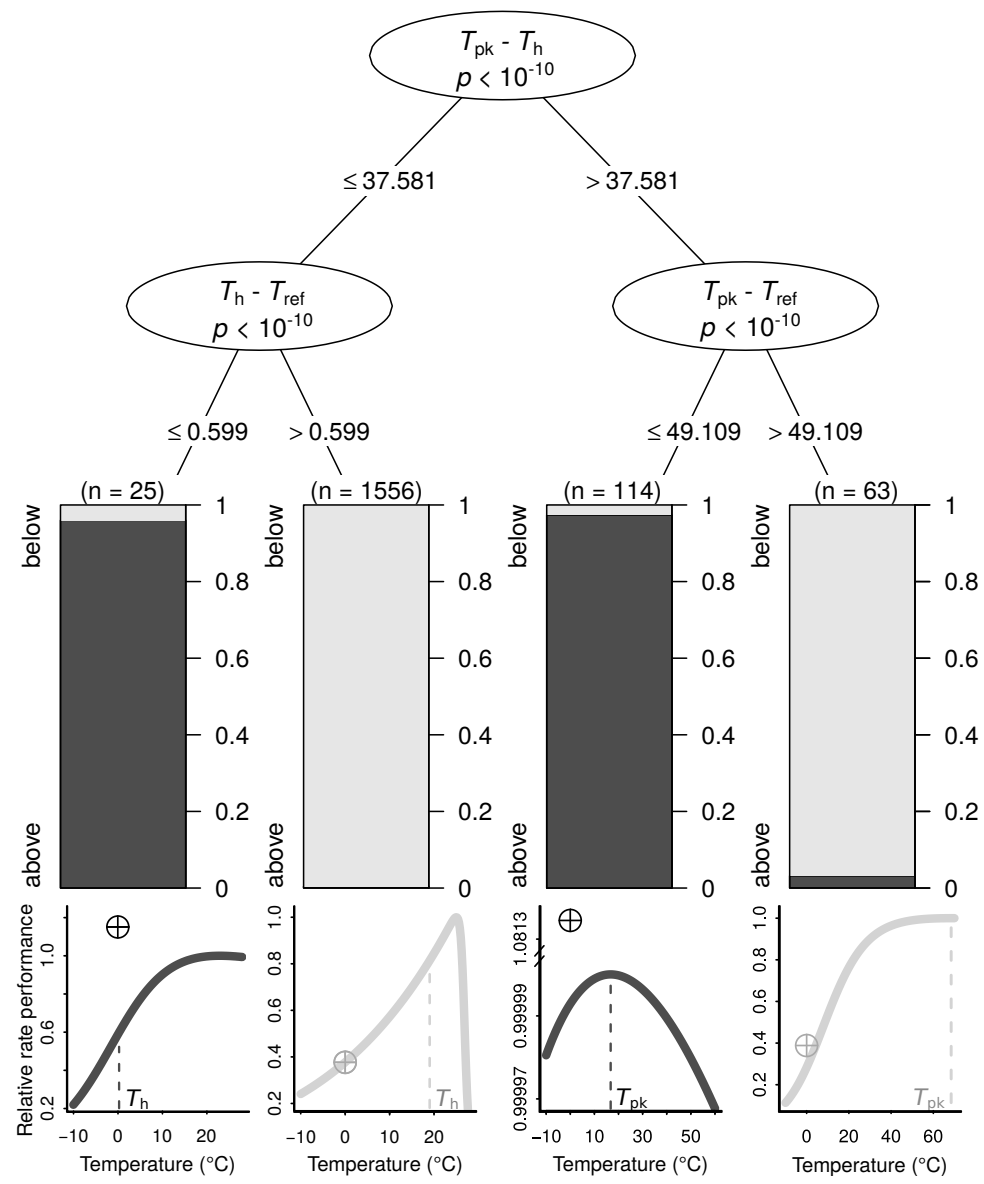

Figure 3: The conditions under which $B_{0}$ is highly overestimated (i.e., $B_{0}>P_{\mathrm{pk}}$; dark grey bars and curves) or less so (i.e., $B_{0}<P_{\mathrm{pk}}$; light grey bars and curves), determined using a conditional inference tree algorithm. Representative examples of thermal performance curves, along with their $B_{0}$ estimates (crossed circles; normalised at $0^{\circ} \mathrm{C}$ for consistency), are shown under each terminal node. The curves are not drawn on the same axes, as their rate performance values vary considerably, even if normalised relatively to the $P_{\mathrm{pk}}$ value of each TPC. For a few very wide - and possibly biologically unrealistic - curves (right half), the difference between $T_{\mathrm{pk}}$ and $T_{\text {ref }}$ determines whether $B_{0}>P_{\mathrm{pk}}$. In contrast, for the remaining curves, a $T_{\mathrm{h}}$ value that is greater than $T_{\text {ref }}$ by more than $0.599^{\circ} \mathrm{C}$ will always lead to $B_{0}$ estimates that are below $P_{\mathrm{pk}}$. 

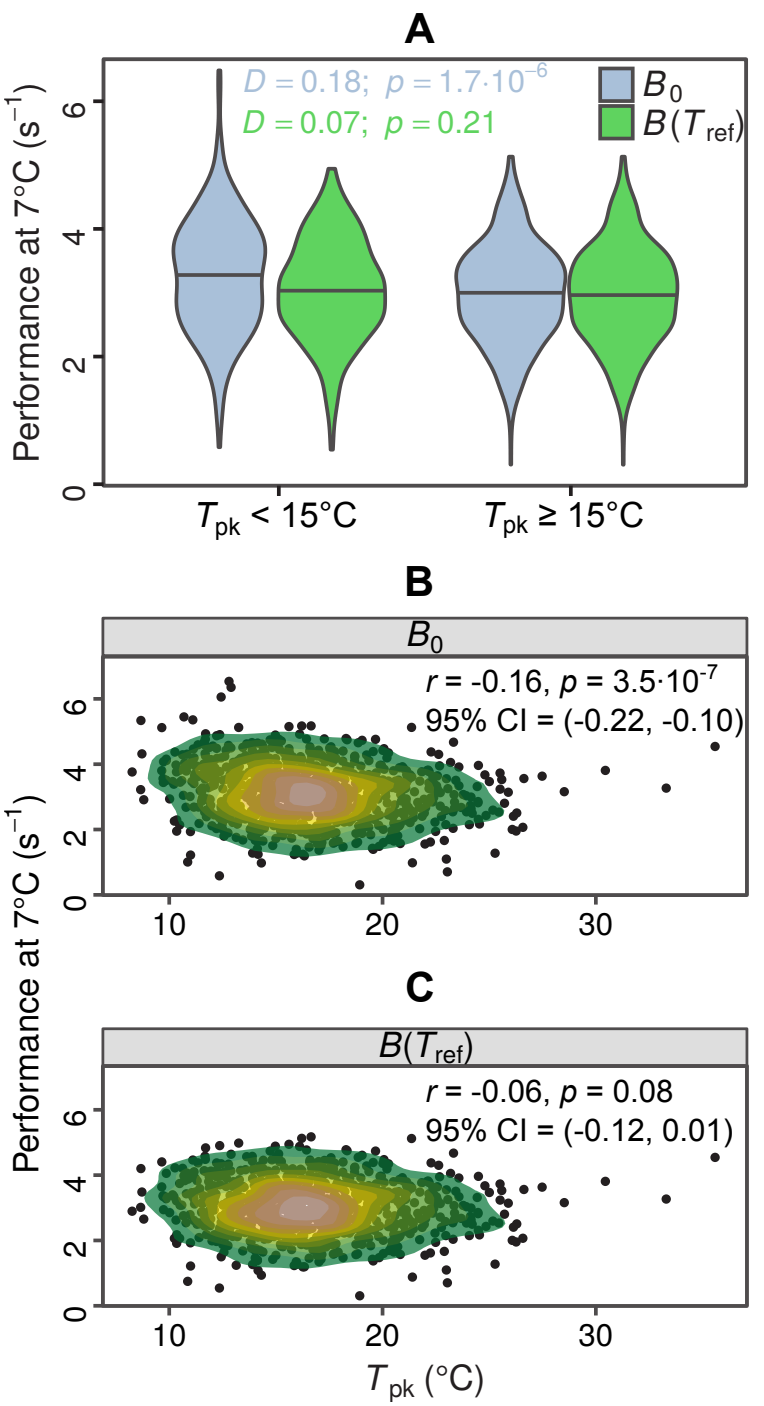

Figure 4: Impacts of exaggerated $B_{0}$ estimates on tests for metabolic cold adaptation. A: Violin plots of rate performance at $T_{\text {ref }}=7^{\circ} \mathrm{C}$, as estimated using $B_{0}$ (light blue) and $B\left(T_{\text {ref }}\right)$ (green), for hypothetical cold-adapted species $\left(T_{\mathrm{pk}}<15^{\circ} \mathrm{C}\right.$; left half $)$ and species adapted to higher temperatures (right half). Horizontal lines indicate the median of each distribution. The statistical significance of the difference in performance between the two temperature groups was evaluated according to the two-sample Kolmogorov-Smirnov test. Based purely on the $B_{0}$ estimates - which get increasingly inflated at low temperatures as $T_{\mathrm{h}}$ approaches $T_{\text {ref }}-$ one would mistakenly conclude that metabolic cold adaptation is present in this dataset. B,C: Correlations of $B_{0}$ with $T_{\mathrm{pk}}$, and $B\left(T_{\text {ref }}\right)$ with $T_{\mathrm{pk}}$. The color surfaces represent the local density of data points. A similar pattern to the previous panel emerges, as the inflated $B_{0}$ estimates - in contrast to the true values - suggest that cold adaptation is present, albeit weakly. 